\title{
Patchwork Stereo: Scalable, Structure-aware 3D Reconstruction in Man-made Environments
}

\author{
Amine Bourki Martin de La Gorce Renaud Marlet Nikos Komodakis \\ LIGM, UMR 8049, Ecole des Ponts, UPE, Champs-sur-Marne, France \\ firstname.lastnamedimagine.enpc.fr
}

\begin{abstract}
In this paper, we address the problem of Multi-View Stereo (MVS) reconstruction of highly regular man-made scenes from calibrated, wide-baseline views and a sparse Structure-from-Motion (SfM) point cloud. We introduce a novel patch-based formulation via energy minimization which combines top-down segmentation hypotheses using appearance and vanishing line detections, as well as an arrangement of creased planar structures which are extracted automatically through a robust analysis of available SfM points and image features. The method produces a compact piecewise-planar depth map and a mesh which are aligned with the scene's structure. Experiments show that our approach not only reaches similar levels of accuracy w.r.t state-of-the-art pixel-based methods while using much fewer images, but also produces a much more compact, structure-aware mesh in a considerably shorter runtime by several of orders of magnitude.
\end{abstract}

\section{Introduction}

Over the last decade, structure-from-motion (SfM) and dense multi-view stereo (MVS) reconstruction have benefited from constant progress in feature detection and matching, and camera calibration, leading to mature systems, e.g, Bundler [36, 35], VisualSFM [41, 42], openMVG [25, 24, 26], PMVS-2 [10], CMP-MVS [14], including consumer products such as Acute3D ContextCapture and Agisoft PhotoScan. Current state-of-the-art methods are now able to produce impressive 3D reconstructions for many scene categories with a rich level of detail, assuming there are enough input images and the scene is sufficiently textured.

However in highly-regular environments such as indoor and outdoor man-made scenes, the complexity of the produced geometry (dense point clouds or meshes) is often detrimental to the structure of reconstructed objects. In such scenes the geometry ubiquitously presents: (i) piecewise planarity, (ii) alignment of objects boundaries with im-

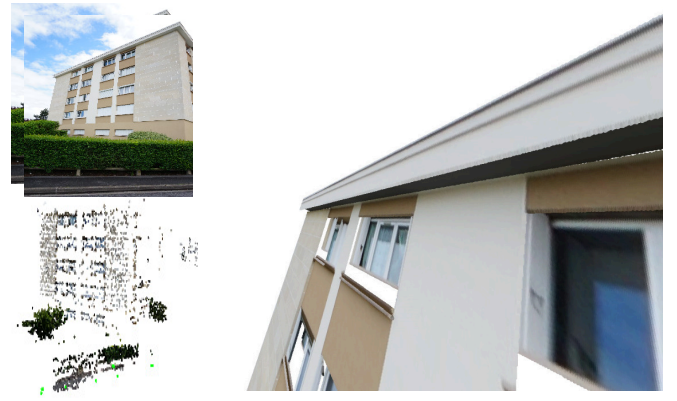

Figure 1: Our method takes a few calibrated images and an SfM point cloud to reconstruct a compact, piecewise-planar mesh aligned with the dominant structure of the scene.

age gradients and (iii) with vanishing directions (VDs), and (iv) surface simplicity, which globally induces planar alignments. This structure is even more difficult to retrieve when only few wide-apart views are considered or available, with broad textureless and specular areas which, altogether, form the typical use-case scenario in urban street-level imagery.

Moreover, the usability of traditional MVS approaches is also limited due to their insufficient computational-andstorage scalability as they consider exhaustive or significant multi-view photoconsistency at the pixel level. Typical runtimes can reach several hours to model a single street, resulting in several millions of polygons and contradicting the paradoxical simplicity of the depicted scenes.

Alternative approaches tackle these issues separately. Superpixel modeling techniques first establish an image partitioning using unsupervised methods $[22,23,3,4]$ to address the problems of robustness and scalability, but fail at respecting structure. Structure-aware reconstruction methods $[9,34]$ on the other hand propagate sparse 2D dominant edge detections and 3D information under heavy regularization and expensive pixelwise computations. A number of restrictive assumptions have been used to simplify the problem, such as a Manhattan-world assumption (MWA) [37, 9], semantic information [18], building footprints [37], hardcoded grammar rules [37] or the additional availability of dense point clouds from laser scans [33,21]. 
In this paper, we address the multi-view reconstruction of structured depth maps from a few images (typically 2-5 wide-baseline images with one reference view) and a sparse SfM point cloud (typically obtained together with image calibration) using a scalable, region-based formulation. In contrast to existing region-based stereo methods, ours does not rely on a bottom-up image partitioning. Rather, we combine vanishing directions, image contours and sparse 3D data to generate top-down segmentation hypotheses, on which we define a Markov Random Field (MRF) topology. The final, structured depth map is retrieved by minimizing a global energy which groups neighboring image patches by enforcing plausible structure-aware connectivities, resulting in a "patchwork" solution.

We demonstrate pixelwise accuracy results on par with state-of-the-art dense MVS pipelines [14] while utilizing much fewer reprojection images and gaining several orders of magnitude in runtime and memory consumption. These improvements are achieved thanks to both our patch-based representation and our robust hypothesis extraction from already-available SfM data. The resulting mesh is compact, and aligned with scenes' structure and image gradients by design, which is achieved with no need of later 3D geometry simplification [29], nor additional complex mesh refinement [39], or tedious primitive fitting steps [19].

Our main contributions are as follows:

- We propose a novel region-based stereo formulation which incorporates structure priors in a principled MRF energy minimization framework where the global energy is amenable to graph-cut inference [5].

- We define a robust joint 2D-3D method for extracting structurally-relevant 2D line and 3D plane hypotheses from principal VDs, image contours and alreadyavailable sparse SfM data. It generates top-down superpixels whose boundaries are aligned with VDs.

- We present an end-to-end pipeline which treats highresolution images (16MP) within a few seconds or minutes per building, paving the way for large-scale, compact, structure-aware urban modeling.

\section{Related Work}

Pixel-level MVS. A number of top-performing general MVS algorithms assume a Delaunay tetrahedralization of an initial 3D point cloud, whose cells are labeled with a discrete occupancy state according to visibility and photometric constraints; the reconstructed surface lies at the interface between empty and non-empty cells [14, 39]. Despite mesh refinement, the resulting surface remains a jagged approximation of a locally-smooth geometry, which may then require expensive post-processing to achieve a compact representation, e.g., by fitting 3D geometric primitives $[19,15]$. The situation is even worse with voxel-based approaches [12, 30]. Pixel-based stereo techniques, which build disparity maps, have seen a tremendous increase in performance since early approaches [16] and their later extensions using second order smoothness priors [40, 27], color models [2] or semantic classification [18]. This category of approaches has been well established for narrowbaseline stereo problems as reported in the Middlebury challenge [32], but it scales poorly in image number and image size; besides, it is sensitive to wider baselines.

Superpixel modeling. Patch-based stereo approaches, e.g., $[22,23,3]$, infer piecewise-planar depth maps for superpixels whose surface is assumed uniform. These superpixels are obtained with unsupervised bottom-up methods, that tend to randomly oversegment highly-textured regions [7] and to produce hexagonal shapes in large homogeneous areas [1]. These methods, in comparison to pixelbased and volumetric approaches, are more scalable and are less sensitive to appearance, viewpoint changes and textureless areas. They are however completely agnostic of the structure of the scene beyond the simple alignment of objects boundaries with image gradients, which translates into many blatant visual artifacts. Bodis-Szomoru et al. [3] build a multi-image graph over superpixels and reconstruct a approximate model which is very well suited for large-scale modeling. However, patch-to-patch stereo matching adds up to the lack of structured boundaries and alignments. It also assumes there are enough SfM points, even in visually homogeneous patches, which often does not hold.

Structure priors. Another line of work models weak structure priors $[34,9]$ by enforcing piecewise-planarity transitions to lie at both strong image gradients and along edges aligned with vanishing directions. However, these are pixelwise approaches and suffer from robustness and scalability issues which restricts their usage to scenes of low complexity and low image resolution $(\leq 3 \mathrm{MP})$. In contrast, our patch-based formulation allows to handle 16MP images with a much lower runtime by several orders of magnitude, without assuming Manhattan scenes [9].

Top-down superpixels. Fouhey et al. [8] use a scene representation relying on multiple top-down partitions of an image. They intersect sets of $2 \mathrm{D}$ rays cast from pairs of vanishing points, defining projective rectilinear superpixels/patches whose boundaries reflects their 3D orientation. The authors use this intermediate representation to estimate the orientation membership of each pixel in a monocular indoor Manhattan-world scene, as well as inter-patch spatial relationships. In contrast, our approach makes use simultaneously (vs. sequentially) of image edge detections, vanishing directions and 3D cues from sparse SfM data to help extract more subtle lines in a robust line-sweep stage.

Mesh alignment. Yet another line of work constructs a mesh in the image domain, and then reconstructs vertices in 3D. Saxena et al. [31] use supervised learning to correlate 
image region appearance with depth information and are able to retrieve a plausible 3D mesh from a single calibrated image for scenes that present a low variation of aspect and structure. Bodis-Szomoru et al. [4] address the problem of $3 \mathrm{D}$ reconstruction from a single image with sparse SfM data by triangulating superpixels [7] in the image domain, and then fitting triangles onto SfM points by penalizing surface curvature. The depth information of triangles with no sparse 3D information is linearly interpolated. This simplifying assumption is made at the expense of geometric accuracy. The rendered reconstructions can be visually satisfactory at a coarse level for nearly flat objects and buildings (e.g., Haussmannian architecture), but cannot model more complex yet ubiquitous elements such as protruding balconies and loggia recesses, especially for patches with low point density. In contrast, our method benefits from sparse SfM cues (where available) and multi-view photoconsistency; it propagates structurally plausible surface associations by favoring planar continuity and crease junctions.

\section{Overview}

Inputs/Outputs. Our method takes a collection of unordered calibrated images (one serving as reference, $\mathcal{I}$, the others for reprojection) and a sparse SfM point cloud $\mathcal{S}$ (given together with calibration information). It produces a structured depth map and a corresponding structured mesh for each reference image. Our notion of structure refers to the following properties w.r.t. the expected output geometry: (i) piecewise-planarity, (ii)+(iii) alignment of object boundaries with strong image gradients and main vanishing directions, (iv) non-local planar and boundary alignments.

Top-down segmentation and 3D plane hypotheses. Our method first computes the dominant VDs visible in $\mathcal{I}$ via a greedy procedure. Top-down superpixels are then generated by creating in $\mathcal{I}$ an arrangement of dominant vanishing lines (VLs). Intuitively, VLs play a key role in capturing the layout of a regular scene as they are plausible indicators of geometric transitions. In order to extract plane candidates consistent with patch boundaries, i.e., to favor crease planar transitions in 3D, VLs and dominant planes must be mutually consistent and aligned. To this end, we extract the 3D hypotheses in a robust vanishing-line-sweeping stage which simultaneously takes into account image features along VLs and sparse 3D data (cf. Section 4).

MRF-Energy minimization. Our energy combines all patches in 3D by enforcing structurally-sound associations in accordance with multi-view patch-wise photoconsistency and SfM cues. It is minimized efficiently (cf. Section 5).

Compact, structured mesh generation. Once the final depth map is recovered, we generate a polygonal mesh for each planar region. This is carried out in the image domain with a 2D Constrained Delaunay Triangulation (CDT) which is then reprojected to 3D (cf. Section 6).

\section{2D Segmentation and 3D Plane Hypotheses}

\subsection{Estimating Vanishing Directions}

As a first step, we extract dominant VDs visible in reference view $\mathcal{I}$. Contrary to [34], we do not merge or cluster them from different images as it would introduce inaccuracies due to calibration imprecision. It could also introduce directions which are irrelevant in the image of interest. We proceed as follows, without MWA, as opposed to [9, 23]:

First, we detect line segments, using LSD [38], and keep the segments with the best scores (lowest $-\log (\mathrm{NFA})$ ). In our experiments, keeping the top 2500 segments of sufficient length (40 pixels), we get enough cues for detecting vanishing points (VPs) with negligible outliers.

Second, we estimate VDs. We use the VP detector of Lezama et al. [20], which handles both Manhattan and nonManhattan cases. As most non-Manhattan architectures yet include 3 Manhattan directions, we first use the Manhattan prior and seek 3 initial Manhattan VDs. We then greedily detect new VDs without the Manhattan prior, putting aside associated lines at each iteration and discarding VDs too close from previous ones $(\leq 5 \mathrm{deg})$, until no more VD is detected. This strategy allows to better retrieve VDs that have subtle sets of supporting evidence. It may yield more than 3 VDs, which may or may not be orthogonal.

\subsection{Dominant Planes}

We extract plane hypotheses in two stages. First, dominant planes are detected from both the VPs and the point cloud $\mathcal{S}$. Next, more subtle planes associated to creases and fine structural details are detected (e.g., window frames).

Concretely, we first discretize the set of plane orientations by considering VP pairs $\vec{v}_{i}, \vec{v}_{j}$ and the associated plane normal $\vec{n}_{i j}$, given the intrinsic calibration matrix $K$ [13]:

$$
\vec{n}_{i j}=\frac{K^{\top} \vec{v}_{i} \times \vec{v}_{j}}{\left\|K^{\top} \vec{v}_{i} \times \vec{v}_{j}\right\|}
$$

Then, for each $\vec{n}_{i j}$, we look for associated plane offsets (signed distance to the camera) that correspond to dominant planes. For this, each point $s \in \mathcal{S}$ votes in a 1D weighted histogram (specific to $\vec{n}_{i j}$ ) in the bin associated to its offset. The weight is $\left|\vec{n}_{i j} . \vec{n}_{s}\right|$ where $\vec{n}_{s}$ is the normal of a plane estimated by PCA analysis from points in a local neighborhood $N(s)$. To limit quantization issues in presence of sparse regions in $\mathcal{S}$, we define $N(s)$ as the ball whose radius is half the distance to the $k$-th nearest neighbor of $s$ [28]. (In our experiments, $k=50$.) The size of a bin is defined as:

$$
g=\min _{i j}\left(\text { median }_{s \in \mathcal{S}}\left(m_{i j}(s)\right)\right)
$$

where $m_{i j}(s)$ is the median of the offsets of points in $N(s)$ w.r.t. $\vec{n}_{i j}$. In our experience, $g$ provides a stable granularity scale throughout different datasets; all dominant planes are retrieved as the maxima of the histogram, unless data is missing, e.g., due to the lack of texture. 


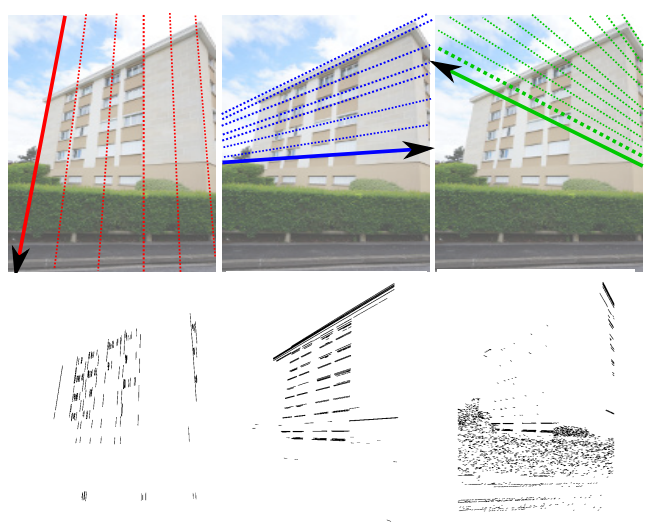

Figure 2: VLs swept from each VP (bottom row). Pixels supporting dominant VLs (top row), based on gradient features.

\subsection{Dominant Vanishing Lines}

We extract dominant VLs in $\mathcal{I}$ as lines with strong and consistent edge information, in the following way.

We first reduce texture sensitivity by applying a bilateral filter ( $\sigma_{r}=130, \sigma_{d}=3$ in experiments). We then filter the image using a Canny-Deriche edge detector [6] with double hysteresis thresholding, resulting in a binary image $\Gamma$. To retrieve more subtle contours, we actually extract edges at multiple image scales $(0.5,0.75,1$ in our implementation) and merge in $\Gamma$ the resulting edge maps with a logical-or.

Then, for each VP, we sweep a VL on the binary edge map. The fixed angular deviation between two successive VLs is the smallest angle among the 4 angles corresponding to 1 pixel of deviation at the 4 image corners. For each swept VL $l$, we consider the rasterized chain of binary pixels it contains. For robustness, we initially apply a 1D Gaussian (with $\sigma=1$ ), re-binarizing the line (with threshold 0.8). For consistency, we only keep as meaningful in $\Gamma$ continuous chains of pixels of length at least 40 . Resulting segments are illustrated on Fig. 2. Finally, dominant VLs are defined as the local maxima of the following score:

$$
\operatorname{dom} V L(l)=\frac{1}{|l|} \sum_{x \in l} \Gamma(x, l)
$$

\subsection{Secondary Lines and Planes}

Leveraging on dominant planes and VL information, we extract more subtle lines and planes. We consider the following three additional cues, based on creaseness.

For each dominant plane $\Pi_{i j}$ with normal $\vec{n}_{i j}$, for each VP $\vec{v}_{k}$ other than $\vec{v}_{i}, \vec{v}_{j}$, and for each VL $l$ swept from $\vec{v}_{i}$ (then symmetrically from $\vec{v}_{j}$ ), we consider a hypothetical plane $\Pi_{i k l}$ defined by the normal $\vec{n}_{i k}$ and the offset s.t. $\Pi_{i k l}$ and $\Pi_{i j}$ intersect in $3 \mathrm{D}$ on a line $L$ which reprojects as $l$. To assess this hypothesis, we measure the following cues:

- $\operatorname{ridge}_{i j k}(l)$ is the number of points in $\mathcal{S}$ that lie in the slice of space at distance at most $g$ of $\Pi_{i k l}$. It is illustrated as the stripe between the green lines in

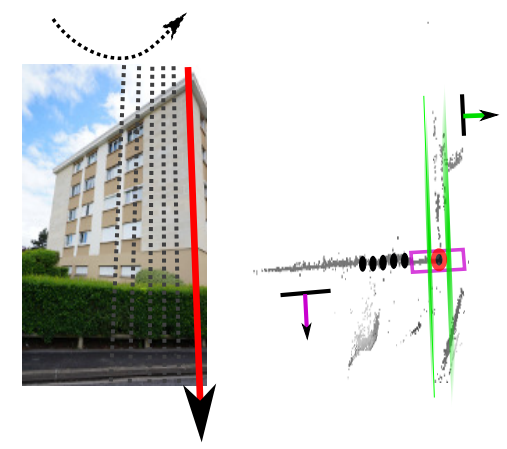

Figure 3: 2D-3D VL sweeping to extract secondary lines and planes (left). Top view of SfM point cloud (right) with regions to measure ridge cues (green), volumic points (red) and plane junctions (purple). Please see text for details.

Fig. 3. As we only want to assess the crease hypothesis at $l$, each point in the slice actually contributes in crease $_{i j k}(l)$ according to its distance $d$ to $L$, with weight $\exp (-d /(40 g))$.

- $\operatorname{volum}_{i j}(l)$ is the number of "volumic" points in $\mathcal{S}$ that lie in a cylinder at distance at most $g$ of $L$. It is illustrated as the disk inside the red circle in Fig. 3. "Volumic" points are considered not to lie on a line or plane, which would not correspond to a crease. The dimensionality of a point $s \in \mathcal{S}$ is given by PCA analysis of neighborhood $N(s)$. It is "volumic" if the 3 largest eigenvalues $e_{1}, e_{2}, e_{3}$ are comparable: $0.35 e_{1} \leq e_{2}, e_{3}$.

- junct $_{i j k}(l)$ is the number of points lying in a rectangular cuboid centered on $L$ with length $8 g$ along $\vec{v}_{j}$ and width $2 g$ along $\vec{v}_{k}$. It is illustrated as the area inside the purple rectangle in Fig. 3. It tells whether dominant plane $\Pi_{i j}$ could have a junction with $\Pi_{i k l}$ at $L$.

Last, if junct $_{i j k}(l) \geq 2$, we consider the following score:

$$
\text { crease }_{i j k}(l)=\operatorname{dom}_{\mathrm{V} L}(l) \text { ridge }_{i j k}(l) \operatorname{volum}_{i j}(l)
$$

The local maxima of crease $_{i j k}(l)$ indicate secondary planes $\Pi_{i j k}$ and vanishing lines $l$.

\subsection{Segmentation into Patches}

The "patchwork", i.e., the final top-down segmentation into patches $p \in \mathcal{P}$, is the 2D arrangement made from dominant and secondary VLs, from which we discard peripheral patches. We only keep patches in the intersection of regions inside the two extreme VLs extracted for each VP. The fact is that peripheral patches often consist of sky, vegetation, ground or clutter pixels, which are not planar. Besides, as not all vanishing orientations are represented at the periphery (in terms of patch boundaries), it could disfavor certain planes during inference, which could propagate by local regularization, altering proper plane assignment.

This simple region clipping automatically restrains the focus of the reconstruction on the main objects of interest. 
It generally defines a convex hull (unless a VP lies in the image). When a convex piecewise-planar structure is observed, this strategy yields a meaningful segmentation, not requiring manual masking [23] nor semantic or planarity classifiers [11]. When it forms a concave region, our assumption still restricts possible detrimental behaviors to the patches that constitute the concave fraction. Our method is however little sensitive to noise and outliers.

\section{Patch-Based Stereo Revisited}

We define a pairwise MRF over the graph $\mathcal{G}=(\mathcal{P}, \mathcal{N})$ where $\mathcal{P}$ is the set of patches in Sect. 4.5 and $\mathcal{N}$ is the neighborhood system of pairs of patches sharing a boundary. Let $\mathcal{L}=\left\{\left(\vec{n}_{1}, d_{1}\right), \ldots,\left(\vec{n}_{N}, d_{N}\right)\right\}$ be the label space of random variables $y_{p} ;\left(\vec{n}_{p}, d_{p}\right)$ represents a plane, uniquely characterized by its normal $\vec{n}_{p}$ and signed offset $d_{p}$.

Our goal is to infer for all patches $p \in \mathcal{P}$ the plane assignment $y_{p}$ with the lowest energy. The energy $E(\mathbf{y})$ encourages planar continuity and crease junctions, over structure disruptions and implausible planar compositions (regularization). It also favors photoconsistency between views at patch level and adherence to the sparse SfM points (data terms). It is defined as follows:

$$
\begin{aligned}
E(\mathbf{y})= & \underbrace{\sum_{p \in \mathcal{P}} w_{p}\left(\Phi_{p}^{\text {Photo }}\left(y_{p}\right)+\Phi_{p}^{3 \mathrm{D}}\left(y_{p}\right)\right)}_{\text {Data terms }} \\
& +\lambda \underbrace{\sum_{(p, q) \in \mathcal{N}} w_{p q} \Psi_{p q}^{\text {Connectivity }}\left(y_{p}, y_{q}\right)}_{\text {Regularization term }}
\end{aligned}
$$

where $\lambda$ balances the contribution of the unary and pairwise potentials, and $w_{p}, w_{p q}$ are adaptive normalizing weights:

$$
w_{p}=\operatorname{area}_{\mathcal{I}}(p) \cdot \exp \left(-\frac{\sigma\left(\mathcal{S}_{p}\right)}{0.1}\right)
$$

where $\operatorname{area}_{\mathcal{I}}(p)$ is the area of $p$, and $\sigma\left(\mathcal{S}_{p}\right)$ is the surface variation of the 3D points reprojecting in $p$, as defined in [28]. This value ranges between 0 (totally planar) and $1 / 3$ (isotropically distributed points).

$$
w_{p q}=|p \sqcap q| \cdot \max \left(0.01, \frac{1}{|p \sqcap q|} \sum_{x \in p \sqcap q} \mu(x)\right)
$$

where $|p \sqcap q|$ is the length of the common edge boundary between $p$ and $q$, and $\mu(x)$ is the edge magnitude at pixel $x$. The different potential functions are detailed below.

\subsection{Data Terms}

Multi-view photoconsistency. $\Phi_{p}^{\text {Photo }}\left(y_{p}\right)$ penalizes appearance dissimilarities between a patch $p$ and its reprojection $\pi_{v}(p)$ in other views $v \in \mathcal{V}$, assuming plane-induced homographies [13]. For regions not reprojecting entirely

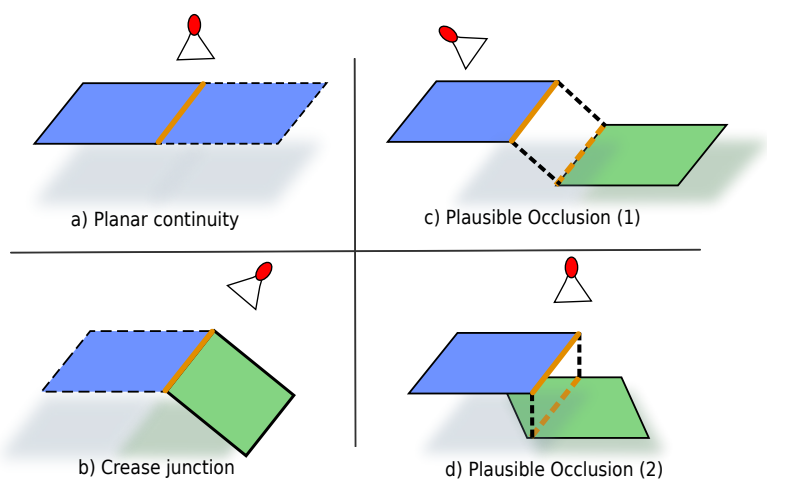

Figure 4: The four pairwise associations modeled by our regularization term. Surface hypotheses are represented with boundaries aligned with a vanishing directions defining their 3D orientations. Best viewed in color.

within $v$, the penalty is a constant. This function is subdivided into an intra-patch photoconsistency and a boundary edge consistency operating on patch boundary pixels $\mathcal{B}_{p}$ and their reprojection $\pi_{v}\left(\mathcal{B}_{p}\right)$.

$$
\Phi_{p}^{\text {Photo }}\left(y_{p}\right)=\frac{1}{|\mathcal{V}|} \sum_{v \in \mathcal{V}}\left\{\alpha \Delta\left(p, \pi_{v}(p)\right)+\beta \mathcal{A}\left(\mathcal{B}_{p}, \pi_{v}\left(\mathcal{B}_{p}\right)\right)\right\}
$$

where $\alpha, \beta$ are model parameters, and $\mathcal{A}(.,$.$) measures the$ proportion of boundary pixels agreeing on the presence of image gradient across views. $\Delta(.,$.$) is a dissimilarity func-$ tion between two image regions related by homography. We consider the zero-mean normalized cross-correlation zncc with exponential normalization for robustness:

where

$$
\Delta\left(p, \pi_{v}(p)\right)=1-\exp \left\{\frac{-\delta^{2}}{0.8}\right\}
$$

$$
\delta=1-\max \left\{0, z n c c\left(p, \pi_{v}(p)\right)\right\}
$$

3D point consistency. We use the sparse $3 \mathrm{D}$ cues to encourage surfaces to fit onto SfM points that reproject within $p$ :

$$
\Phi_{p}^{3 \mathrm{D}}\left(y_{p}\right)=1-\exp \left\{\frac{-\phi^{2}}{0.3}\right\}
$$

where

$$
\phi=\frac{\gamma}{\tau .\left|\mathcal{S}_{p}\right|} \sum_{s \in \mathcal{S}_{p}} \min \left(\tau, \frac{\mathcal{D}\left(s, y_{p}\right)}{g}\right)
$$

where $\gamma$ is a model parameter, $\mathcal{S}_{p}$ is the subset of SfM points reprojecting within $p, \tau$ is a distance threshold (measured in $g$ units), and $\mathcal{D}\left(s, y_{p}\right)$ is the point-to-plane 3D distance.

\subsection{Regularization}

Representing 3D orientations by using vanishing points (Eq. (1)) suggests that two planar surfaces oriented resp. towards $\vec{n}_{i j}$ and $\vec{n}_{i j^{\prime}}$ are likely to intersect in the image plane 


\begin{tabular}{|c|c|c|c|c|c|c|c|c|c|c|}
\hline & & & & PMVS-2 [10] & CMP-MVS[14] & \multicolumn{5}{|c|}{ Patchwork Stereo (PWS = ours) } \\
\hline Scenes & \#Img & $\# \mathrm{SfM} / \mathcal{I}$ & Resol. & \#Points & \#Triangles & \#Reproj.views & \#VDs & \#Normals & \#Planes & \#Patches \\
\hline Bry2 & 31 & 2081 & $16 \mathrm{MP}$ & 470185 & 587220 & 2 & 3 & 3 & 45 & 2003 \\
\hline AugusteC & 11 & 2980 & $16 \mathrm{MP}$ & 498270 & 653228 & 3 & 3 & 3 & 78 & 2980 \\
\hline GMU [23] & 5 & 578 & $2 \mathrm{MP}$ & 25750 & 23057 & 3 & 3 & 3 & 28 & 1416 \\
\hline Lecourbe 1 & 23 & 5135 & $16 \mathrm{MP}$ & 603368 & 423854 & 3 & 3 & 3 & 177 & 6048 \\
\hline Hameau & 36 & 21824 & $16 \mathrm{MP}$ & 766744 & 1143405 & 3 & 3 & 3 & 89 & 5351 \\
\hline
\end{tabular}

Table 1: Datasets and comparative reconstruction characteristics. \#SfM/I : \# of SFM points reprojecting on $\mathcal{I}$.

at a crease edge $\vec{e}_{p q}$ (in orange in Figure 4) aligned with the common vanishing direction $\vec{v}_{i}$. Our pairwise regularization prior $\Psi_{p q}^{\text {Connectivity }}\left(y_{p}, y_{q}\right)$ relies on this assumption by reasoning on the connectivity of neighboring patches and imposing a preference over the possible configurations:

$$
\begin{aligned}
& \Psi_{p q}^{\text {Connectivity }}\left(y_{p}, y_{q}\right) \\
& = \begin{cases}0 & : \text { if } y_{p}=y_{q},\left(y_{p}, y_{q}\right) \in \mathcal{T}_{\text {continuity }} \\
\lambda_{1} & : \text { elseif }\left(y_{p}, y_{q}\right) \in \mathcal{T}_{\text {crease }} \\
\lambda_{2} & : \text { elseif }\left(y_{p}, y_{q}\right) \in \mathcal{T}_{\text {occlusion }_{1}} \\
\lambda_{3} & : \text { elseif }\left(y_{p}, y_{q}\right) \in \mathcal{T}_{\text {occlusion }_{2}} \\
\lambda_{4} & : \text { otherwise }\end{cases}
\end{aligned}
$$

where $0 \leq \lambda_{1} \leq \lambda_{2} \leq \lambda_{3} \leq \lambda_{4}$ are the respective costs for neighboring patches, and $\left(y_{p}, y_{q}\right) \in \mathcal{T}_{\text {continuity }}$ lie on the same plane, $\left(y_{p}, y_{q}\right) \in \mathcal{T}_{\text {crease }}$ form a crease junction, $\left(y_{p}, y_{q}\right) \in \mathcal{T}_{\text {occlusion }_{1}}$ lie at a depth discontinuity where $\vec{e}_{p q}$ is consistent with the orientations of both $p$ and $q$, and $\left(y_{p}, y_{q}\right) \in \mathcal{T}_{\text {occlusion }_{2}}$ are such that $\vec{e}_{p q}$ is consistent only with the occluding (fronting) patch, as illustrated in Fig. 4. All other configurations are given a prohibitive penalty $\lambda_{4}$.

Depending on how these penalties are set, the smoothness function can either be a metric, or a semi-metric.The metric case allows more efficient inference as it guarantees the solution to be at a known factor from global optimum, but is more restrictive in expressive power [17].

\section{Structure-Aware Mesh Generation}

After inferring a plane for each patch, our structured planemap representation contains a number of polygons per reconstructed plane. For each plane, we merge all associated polygons, producing larger but fewer polygons, possibly with holes. By construction, patches are either adjacent one to another or disjoint, which simplifies merging. By construction also, the polygon boundaries are aligned with VDs and image gradients. A 2D triangle mesh for these merged polygons can be then produced using a constrained Delaunay Triangulation, and then lifted to 3D.

\section{Evaluation}

We evaluate our approach on 5 challenging datasets of individual buildings presenting textureless areas and repetitive patterns, for which we use only a few wide-baseline images. Statistics for each dataset are given in Table 1. All experiments use the same parameters: $\alpha=1, \beta=0.4$, $\gamma=0.5, \lambda=30, \lambda_{1}=0, \lambda_{2}=0.6, \lambda_{3}=3.8, \lambda_{4}=50$.

Quantitative results. We quantify pixelwise accuracy of our reconstructions w.r.t. a reference mesh built with CMPMVS [14] and two point clouds built using PMVS-2 with and without Poisson surface reconstruction [10]. For these baselines, we use all of the available images of each scene.

Fig. 5 shows, for each row, the reference image of each dataset, the corresponding top-down segmentation, a qualitative view of the output $3 \mathrm{D}$ model and the corresponding quantitative results per scene. For each method, in the right column, we vary the tolerated error as a fraction of the scene's depth range and accumulate the proportion of correctly reconstructed pixels (up to the given tolerance) w.r.t. the reference mesh; the higher the curve, the better the performance. This figure shows the following: (i) The sparse PMVS-2 method has poor overall accuracy due to the lack of reconstructed points in wide textureless areas. (ii) Its dense counterpart (PMVS-2+Poisson) performs better than our model (PWS) and its ablated versions for AugusteC and Hameau, which is explained by the significant amount of additional images. (iii) For the GMU dataset, PWS has a higher curve, which is due to the lack of images for CMPMVS and PMVS-2+Poisson (only 5). (iv) In Bry2, the performance of PWS is on par with the baseline.

Although using only a small subset of wide-baseline views, our method (PWS) achieves comparable accuracy results while providing a much more compact geometry which respects the structural regularity of the scene.

Ablative study. Fig. 5 also shows results with ablated variants of our data terms, to assess their importance. (When cancelling a term, we make sure the relative weights of the data and regularization terms stay the same.)

Keeping only the SFM term $\Phi_{p}^{3 \mathrm{D}}\left(y_{p}\right)$ sometimes leads to severe errors. This robustness issue corresponds to a few anomalous planes due to point could sparsity. Apart from SFM, PWS is comparable to its ablated models, sometimes slightly better in terms of pixelwise accuracy. However, it is difficult to see quantitatively the difference because of the relative lack of accuracy of the CMP-MVS reference. Still, a qualitative analysis, as illustrated in Fig. 6, shows that the full PWS model presents a much more regular, structured appearance and is visually more pleasing. 

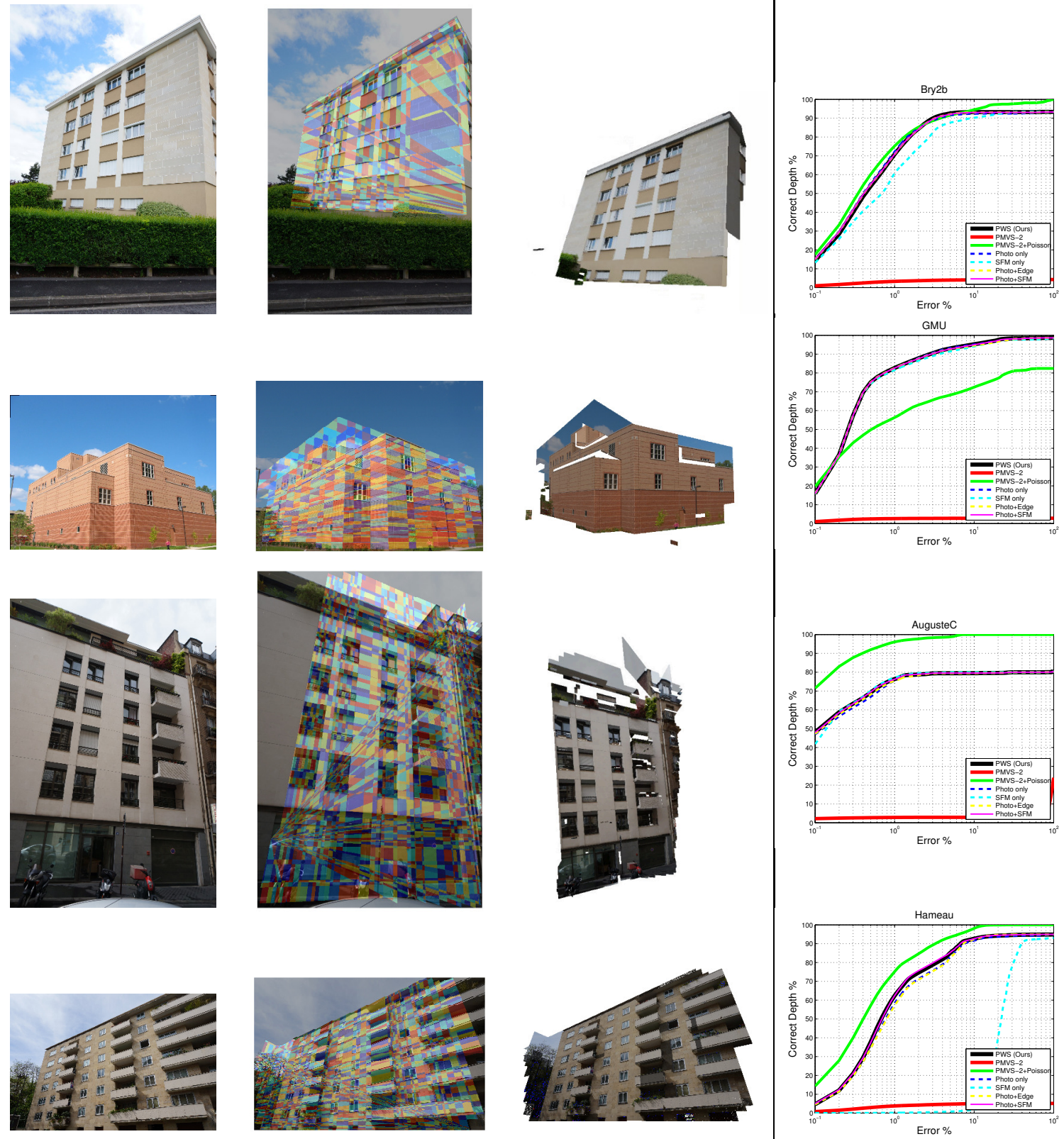

Figure 5: Each row illustrates a different dataset. In columns, from left to right: (i) reference view, (ii) our segmentation, (iii) our 3D reconstruction, (iv) semi-log-scale accuracy w.r.t CMP-MVS reference mesh. We plot the proportion of pixels whose depth is correct up to a given error tolerance, expressed as a fraction of the scene's thickness (labeled Error \%). We compare with PMVS-2 [10] and different ablations of our data terms. Best viewed in color.

Indicative runtime. Our CPU implementation is a mixture of pure vectorized Matlab / Mex / C++. The two main computational bottlenecks of our method are the multiview photoconsistency, which is computed for all patches through all planar hypotheses, and the pairwise costs. Both of these tasks are written in vectorized pure Matlab, and the photoconsistency could benefit from significant speed-ups.

Photoconsistency runs in roughly 1s per 16MP image per plane candidate on a modest laptop with an Intel Core2Duo
2.40Ghz, 4GB RAM. Other running times are negligible.

Comparison to related work. [4] provides quantitative results on scenes for which our VD-based segmentation does not make sense, e.g. arches and columns of Herz-Jesu. Only scenes of streets M, P, Z of Mirbel (low-resolution, $<1 \mathrm{MP}$ images) are relevant to us, but are unknown subsets of the ETHZ RueMonge 2014 dataset. The reference (highresolution) mesh is unavailable anyway. Still, we ran our method, with only 2 reprojection views, on a RueMonge fa- 

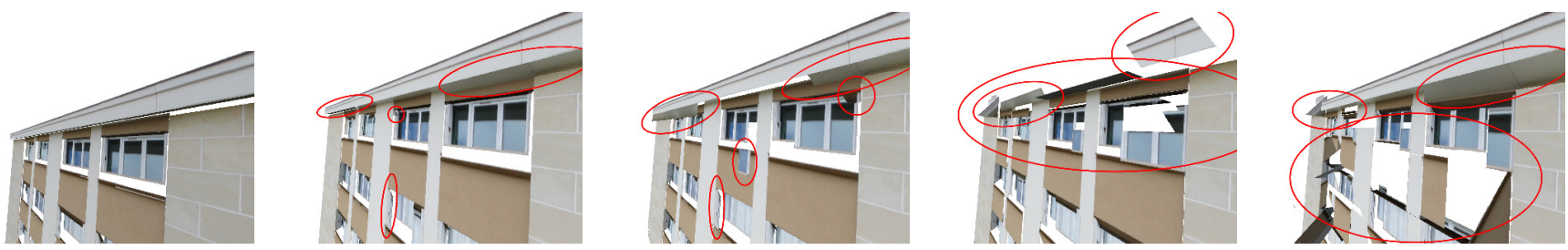

Figure 6: Qualitative comparison of different ablations of our data terms. From left to right: (i) our full model, (ii) Photo+SFM, (iii) Photo only, (iv) Photo+Edge, (v) SFM only. Even though the global pixelwise accuracy may be comparable between different truncated versions of our model (cf. Fig. 5), removing data terms translates into noticeable artifacts which degrade the 3D structure through erroneous depth or even surface orientations. Best viewed in color.
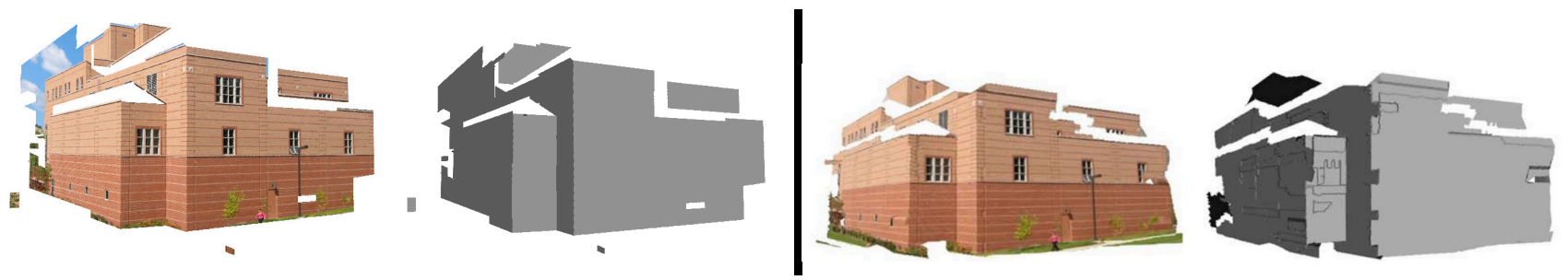

Figure 7: Side-by-side comparison with prior work Superpixel Stereo (SPS) [23]. On the left side, our reconstruction presents sharp edges and perfect crease transitions, seamless plane continuity and the alignment of surface boundaries with VDs and image contours. On the right side, SPS [23] presents an uneven planar geometry along flat surfaces and patches tend to straddle between different plane orientation at crease transitions, as they are agnostic of VDs. Best viewed in color.

cade looking like Fig. 1,3,6 in [4]. Our reconstruction (cf. Fig. 8) is better aligned with the structure: window and balcony edges are straighter and sharper. Besides, we have much less triangles per image ( $<680$ vs $15 \mathrm{k}$ ). [9, 23, 34] do not provide any quantitative evaluation of accuracy; in any case, they do not address both structure and scalability, as we do. Comparing with [23], our result is much better, as illustrated on Fig. 7. Our junctions form perfect creases. Our misreconstructed patches correspond either the sky or to regions occluded in other views. All our patches are perfectly aligned with VDs in contrast to patches in [23] which form arbitrary shapes and do not touch in 3D.

As for speed, [4] processes on average 1 view of $1 \mathrm{MP}$ per $2 s$ and a facade in RueMonge is seen by about 10 views, yielding a rate of about 20s/MP/facade. With Matlab, we process 1 plane hypothesis for a 16MP image in about 1s; assuming 80-plane scenes with 3 reprojection views per facade, our rate is $80 * 3 * 1 / 16=15 \mathrm{~s} / \mathrm{MP} /$ facade, comparable to [4]. Likewise, [9] takes more than 300s/MP/facade and [34] takes 60s/MP/image for scenes with 11-61 images. [23] does not provide complete time information.

\section{Conclusion}

We have presented a novel approach for automatic multiview reconstruction of structured depth maps from only a few, wide-baseline high-resolution photographs. Our method produces compact meshes which are aligned with the dominant structural traits of the scene (vanishing directions and edges). We have shown how top-down segmentation hypotheses and sparse 3D data can capture most of non-local planar alignments which are typical of man-made scenes. Working at the patch-level allows significant improvements in robustness and scalability without any loss of information w.r.t working on individual pixels. This paves the way for large-scale structure-aware urban modeling with plausible, visually pleasing digital rendering.

\section{Acknowledgements}

This work was partly supported by ANR project Semapolis ANR-13-CORD-0003.
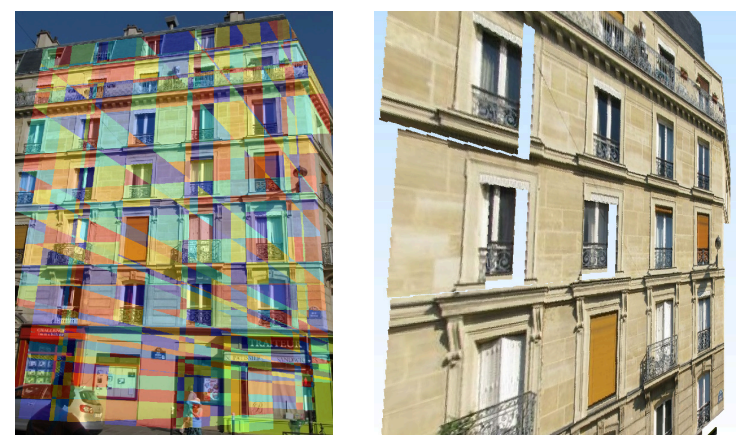

Figure 8: Segments \& planes of Haussmannian facade. 


\section{References}

[1] R. Achanta, A. Shaji, K. Smith, A. Lucchi, P. Fua, and S. Susstrunk. SLIC superpixels compared to state-of-the-art superpixel methods. IEEE Transactions on Pattern Analysis and Machine Intelligence (PAMI), 34(11):2274-2282, 2012.

[2] M. Bleyer, C. Rother, P. Kohli, D. Scharstein, and S. Sinha. Object stereo - joint stereo matching and object segmentation. In IEEE Conference on Computer Vision and Pattern Recognition (CVPR), pages 3081-3088, 2011.

[3] A. Bodis-Szomoru, H. Riemenschneider, and L. V. Gool. Fast, approximate piecewise-planar modeling based on sparse structure-from-motion and superpixels. In IEEE Conference on Computer Vision and Pattern Recognition (CVPR), pages 469-476, 2014.

[4] A. Bódis-Szomorú, H. Riemenschneider, and L. Van Gool. Superpixel meshes for fast edge-preserving surface reconstruction. In IEEE Conference on Computer Vision and Pattern Recognition (CVPR), 2015.

[5] Y. Boykov, O. Veksler, and R. Zabih. Fast approximate energy minimization via graph cuts. IEEE Transactions on Pattern Analysis and Machine Intelligence (PAMI), 23(11):1222-1239, 2001.

[6] R. Deriche. Using Canny's criteria to derive a recursively implemented optimal edge detector. International Journal of Computer Vision (IJCV), 1(2):167-187, 1987.

[7] P. F. Felzenszwalb and D. P. Huttenlocher. Efficient graphbased image segmentation. International Journal of Computer Vision (IJCV), 59(2):167-181, 2004.

[8] D. F. Fouhey, A. Gupta, and M. Hebert. Unfolding an indoor origami world. In European Conference on Computer Vision (ECCV), pages 687-702. Springer, 2014.

[9] Y. Furukawa, B. Curless, S. M. Seitz, and R. Szeliski. Manhattan-world stereo. In IEEE Conference on Computer Vision and Pattern Recognition (CVPR), pages 1422-1429, 2009.

[10] Y. Furukawa and J. Ponce. Accurate, dense, and robust multiview stereopsis. IEEE Transactions on Pattern Analysis and Machine Intelligence (PAMI), 32(8):1362-1376, 2010.

[11] D. Gallup, J.-M. Frahm, and M. Pollefeys. Piecewise planar and non-planar stereo for urban scene reconstruction. In IEEE Conference on Computer Vision and Pattern Recognition (CVPR), pages 1418-1425, 2010.

[12] C. Häne, C. Zach, A. Cohen, R. Angst, and M. Pollefeys. Joint 3D scene reconstruction and class segmentation. In IEEE Conference on Computer Vision and Pattern Recognition (CVPR), 2013.

[13] R. Hartley and A. Zisserman. Multiple view geometry in computer vision, volume 2. Cambridge Univ Press, 2000.

[14] M. Jancosek and T. Pajdla. Multi-view reconstruction preserving weakly-supported surfaces. In IEEE Conference on Computer Vision and Pattern Recognition (CVPR), pages 3121-3128, 2011.

[15] N. Kobyshev, H. Riemenschneider, A. Bódis-Szomorú, and L. Van Gool. Architectural decomposition for 3D landmark building understanding. In IEEE Winter Conference on Applications of Computer Vision (WACV), 2016.
[16] V. Kolmogorov and R. Zabih. Computing visual correspondence with occlusions using graph cuts. In IEEE International Conference on Computer Vision (ICCV), volume 2, pages 508-515, 2001.

[17] V. Kolmogorov and R. Zabin. What energy functions can be minimized via graph cuts? IEEE Transactions on Pattern Analysis and Machine Intelligence (PAMI), 26(2):147-159, 2004.

[18] L. Ladickỳ, P. Sturgess, C. Russell, S. Sengupta, Y. Bastanlar, W. Clocksin, and P. H. Torr. Joint optimization for object class segmentation and dense stereo reconstruction. International Journal of Computer Vision (IJCV), 100(2):122-133, 2012.

[19] F. Lafarge, R. Keriven, M. Brdif, and H.-H. Vu. A hybrid multiview stereo algorithm for modeling urban scenes. IEEE Transactions on Pattern Analysis and Machine Intelligence (PAMI), pages 5-17, 2013.

[20] J. Lezama, R. G. v. Gioi, G. Randall, and J.-M. Morel. Finding vanishing points via point alignments in image primal and dual domains. In IEEE Conference on Computer Vision and Pattern Recognition (CVPR), pages 509-515, 2014.

[21] Y. Li, Q. Zheng, A. Sharf, D. Cohen-Or, B. Chen, and N. J. Mitra. 2D-3D fusion for layer decomposition of urban facades. In IEEE International Conference on Computer Vision (ICCV), pages 882-889, 2011.

[22] B. Mičušík and J. Košecká. Piecewise planar city 3D modeling from street view panoramic sequences. In IEEE Conference on Computer Vision and Pattern Recognition (CVPR), pages 2906-2912, 2009.

[23] B. Mičušík and J. Košecká. Multi-view superpixel stereo in urban environments. International Journal of Computer Vision (IJCV), 89(1):106-119, 2010.

[24] P. Moulon, P. Monasse, and R. Marlet. Adaptive structure from motion with a contrario model estimation. In Asian Conference on Computer Vision (ACCV), pages 257-270, 2012.

[25] P. Moulon, P. Monasse, and R. Marlet. OpenMVG (open multiple view geometry), 2012. https: / / git hub. com/ openMVG.

[26] P. Moulon, P. Monasse, and R. Marlet. Global fusion of relative motions for robust, accurate and scalable structure from motion. In IEEE International Conference on Computer Vision (ICCV), pages 3248-3255, 2013.

[27] C. Olsson, J. Ulén, and Y. Boykov. In defense of 3D-label stereo. In IEEE Conference on Computer Vision and Pattern Recognition (CVPR), pages 1730-1737, 2013.

[28] M. Pauly, M. Gross, and L. P. Kobbelt. Efficient simplification of point-sampled surfaces. In Proc. of the conference on Visualization, pages 163-170. IEEE Computer Society, 2002.

[29] D. Salinas, F. Lafarge, and P. Alliez. Structure-aware mesh decimation. Computer Graphics Forum, 34(6):211-227, 2015.

[30] N. Savinov, C. Hane, M. Pollefeys, et al. Discrete optimization of ray potentials for semantic $3 \mathrm{D}$ reconstruction. In IEEE Conference on Computer Vision and Pattern Recognition (CVPR), pages 5511-5518, 2015. 
[31] A. Saxena, M. Sun, and A. Y. Ng. Make3D: Learning 3D scene structure from a single still image. IEEE Transactions on Pattern Analysis and Machine Intelligence (PAMI), 31(5):824-840, 2009.

[32] D. Scharstein and R. Szeliski. A taxonomy and evaluation of dense two-frame stereo correspondence algorithms. International Journal of Computer Vision (IJCV), 47(1-3):7-42, 2002.

[33] C.-H. Shen, S.-S. Huang, H. Fu, and S.-M. Hu. Adaptive partitioning of urban facades. ACM Transactions on Graphics (TOG), 30(6):184, 2011.

[34] S. N. Sinha, D. Steedly, and R. Szeliski. Piecewise planar stereo for image-based rendering. In IEEE International Conference on Computer Vision (ICCV), pages 1881-1888, 2009.

[35] N. Snavely. Bundler: Structure from motion (SfM) for unordered image collections, 2010. v0.4, http: / / www. cs . cornell.edu/ \verb+ +snavely/bundler.

[36] N. Snavely, S. Seitz, and R. Szeliski. Photo tourism: Exploring image collections in 3D. ACM Transactions on Graphics (TOG), 25(3):835-846, July 2006.

[37] C. A. Vanegas, D. G. Aliaga, and B. Benevs. Building reconstruction using Manhattan-world grammars. IEEE Conference on Computer Vision and Pattern Recognition (CVPR), 0:358-365, 2010.

[38] R. G. von Gioi, J. Jakubowicz, J.-M. Morel, and G. Randall. LSD: a fast line segment detector with a false detection control. IEEE Transactions on Pattern Analysis and Machine Intelligence (PAMI), 32(4):722-732, 2008.

[39] H.-H. Vu, P. Labatut, J.-P. Pons, and R. Keriven. High accuracy and visibility-consistent dense multiview stereo. IEEE Transactions on Pattern Analysis and Machine Intelligence (PAMI), 34(5):889-901, 2012.

[40] O. Woodford, P. Torr, I. Reid, and A. Fitzgibbon. Global stereo reconstruction under second-order smoothness priors. IEEE Transactions on Pattern Analysis and Machine Intelligence (PAMI), 31(12):2115-2128, 2009.

[41] C. Wu. VisualSFM: A visual structure from motion system, 2011. http: / / ccwu. me/vsfm.

[42] C. Wu. Towards linear-time incremental structure from motion. In IEEE International Conference on $3 D$ Vision (3DV), pages 127-134, 2013. 